hep-th/9606174, VPI-IPPAP-96-3

\title{
Invariant regularization of anomaly-free chiral theories
}

\author{
Lay Nam Chang * \\ and \\ Chopin Soo ${ }^{\dagger}$ \\ Department of Physics, and \\ Institute for Particle Physics and Astrophysics, \\ Virginia Tech, \\ Blacksburg, VA 24061-0435, U.S.A. \\ PACS number(s): 11.15.-q, 11.30.Rd, 04.62.+v
}

We present a generalization of the Frolov-Slavnov invariant regularization scheme for chiral fermion theories in curved spacetimes. The Lagrangian level regularization is explicitly invariant under all the local gauge symmetries of the theory, including local Lorentz invariance. The perturbative scheme works for arbitrary representations which satisfy the chiral gauge anomaly and the mixed Lorentz-gauge anomaly cancellation conditions. Anomalous theories on the other hand manifest themselves by having divergent fermion loops which remain unregularized by the scheme. Since the invariant scheme is promoted to also include local Lorentz invariance, spectator fields which do not couple to gravity cannot be, and are not, introduced. Furthermore, the scheme is truly chiral (Weyl) in that all fields, including the regulators, are left-handed; and only the left-handed spin connection is needed. The scheme is, therefore, well suited for the study of the interaction of matter with all four known forces in a completely chiral fashion. In contrast with the vectorlike formulation, the degeneracy between the Adler-Bell-Jackiw current and the fermion number current in the bare action is preserved by the chiral regularization scheme.

${ }^{\dagger}$ Electronic address: soo@theory.uwinnipeg.ca. Present address:Dept. of Physics, University of Winnipeg, Winnipeg, Manitoba, Canada R3C 2E9. *Electronic address: laynam@vt.edu 


\section{INTRODUCTION}

It is believed that the existence of an invariant regularization for a quantum field theory of chiral fermions is predicated upon the absence of perturbative anomalies. It is interesting to ask whether an explicitly invariant regularization scheme for chiral fermions can be constructed so that it successfully regularizes the theory when the representation of the chiral fermion multiplet is anomaly free, and fails to do so precisely when the representation is not. In the following, we present a scheme of regularization at the Lagrangian level incorporating this feature, which is suitable for describing chiral theories in curved spacetimes.

Frolov and Slavnov [1] first proposed an explicitly gauge invariant regularization which makes essential use of an infinite tower of Pauli-Villars-Gupta regulators [2]. The theory was originally based on the $\mathrm{SO}(10)$ multiplet [3] - or rather, the 16-dimensional chiral representation of $\operatorname{Spin}(10)$ - with the Standard Model embedded in it. Since invariant regulator mass terms are required for this type of Pauli-Villars-Gupta regularization, it is necessary to "double" in internal gauge group space by also including fields which transform according to the complex conjugate representation [4]. The tower of regulators is therefore neutral with respect to $\Gamma_{11}$, and can regularize only the singlet part of the $\frac{1}{4}\left(1-\Gamma_{11}\right)\left(1-\gamma^{5}\right)$ projection of the bare gauge current. However, it is shown that the $\Gamma_{11}$ part gives rise to no further divergences due to the fact that the trace of four or less generators of $\operatorname{Spin}(10)$ with $\Gamma_{11}$ vanishes. Frolov and Slavnov also proposed a discretization of the theory [5]. It is believed that the Nielsen-Ninomiya no-go theorem [6 is surmounted by the presence of the infinite tower of regulator fields. It was not immediately clear from this discussion if the method generalizes to arbitrary anomaly-free chiral theories, although it was clear how to regularize theories based upon $\mathrm{SO}(2 n \geq 10)$ groups. Okuyama and Suzuki[7] clarified and generalized the original Frolov-Slavnov idea to include fermion multiplets in arbitrary real and pseudoreal representations. But the generalization to curved spacetimes

\footnotetext{
${ }^{1}$ This does not necessarily mean that some version of the theory cannot be defined. For instance, the anomalous chiral Schwinger model can be solved exactly. However, the gauge invariance of the theory is lost.

${ }^{2}$ Chirality in Spin(10) is defined relative to $\Gamma_{11}$, which is proportional to the product of all ten Dirac matrices spanning the Clifford algebra in ten dimensional Euclidean space.
} 
and Abelian gauge groups remained somewhat unclear.

The scheme was considered in a different light by Fujikawa[8], and by Narayanan and Neuberger 99. They doubled in external or Lorentz space by including right-handed as well as left-handed regulator fields. In these vectorlike formulations, no doubling in the internal symmetry group is needed. To study nonperturbative effects, Narayanan and Neuberger 10 also proposed the "overlap formalism" by treating the extra index associated with the tower of regulators as an additional dimension of spacetime, and by defining the chiral fermion determinant as the overlap of two different gound states of the higher dimensional theory. As such, the Nielsen-Ninomiya no-go theorem of putting chiral fermions on a lattice may be overcome by treating the chiral theory in $2 n$ dimensions as the target of another in $2 n+1$ dimensions in which there is no concept of chirality.

In these vectorlike formulations, the tower is parity even or $\gamma^{5}$ neutral. So doubling in external space by including right-handed fields now regularizes the left-right symmetric part, leaving the $\gamma^{5}$ part of the bare gauge current untouched. For anomaly-free theories, it can be argued that there are no divergent parity-odd diagrams generated by the gauge current. As a result, the gauge current is regularized for arbitrary, perturbatively anomaly-free representations. However, as noted by Fujikawa [8], the parity-nonconserving amplitude with gauge singlet currents can be divergent. For instance, the fermion number current is not free of divergences, and is different from the axial or Adler-Bell-Jackiw(ABJ) current at the regularized level[8]. On the other hand, in the totally left-handed Frolov-Slavnov formulation, the degeneracy between the well-defined ABJ singlet current and the fermion number current in the original bare action is preserved by the chiral regularization scheme.

What happens when the Frolov-Slavnov regularization scheme is promoted to include local Lorentz invariance and the effects of curved spacetimes? Two issues immediately arise. First, it is known that chiral fermions in curved spacetimes can introduce a further perturbative mixed Lorentzgauge anomaly[11]. Second, all fields couple to gravity, and the trick of introducing spectators inherent in some methods of defining chiral theories may not work [0, 12]. For example, in Ref[1], the Standard Model (or any anomaly-free subgroup of $\mathrm{SO}(10)$ ) can be recovered by taking the gauge field $W_{\mu}$ to lie only in the relevant subgroup. However, if the regularization is extended to include invariance under local Lorentz transformations, then the 
extra spectator "neutrino," which is not coupled to any internal gauge field, becomes physical as a result of its coupling to gravity.

Similar remarks apply to right-handed fermions. These make an appearance in a chiral theory either as regulators, as in vectorlike schemes, or as spectators in defining propagators [7]. All these fields get coupled to gravity and become physically interacting degrees of freedom. The key point is that there can be no passive spectators if the regularization scheme is promoted to also respect local Lorentz invariance.

There is yet another issue we need to be aware of. Right-handed multiplets can be introduced in a covariant way for curved spacetimes only if one also allows for right-handed spin connections. It is known through the work of Ashtekar and others 13, 14] that the (anti-)self-dual formulation of gravity which involves only the left-handed spin connection, rather than the full spin connection, may provide a complete description of gravity in four dimensions. These right-handed spin connections are generally not independent of the left-handed ones 13, and their presence might therefore complicate the gravity field equations unnecessarily.t. Indeed in the (anti-)self-dual formulation, no right-handed fermions should be introduced [15, 16].

For these reasons, we examine in this paper a regularization scheme that is based only upon left-handed fields, with no spectators. This scheme extends the Frolov-Slavnov regularization to anomaly-free chiral theories in arbitrary complex representations in curved spacetimes. The regularized chiral fermion action is explicitly gauge, Lorentz, and diffeomorphism invariant, and is truly chiral (Weyl) in the sense that only left-handed spin connections and left-handed multiplets are introduced. The proposed regularization is therefore well suited for the study of the interaction of matter with all the four known forces in a completely chiral manner 16.

We shall show that the generalization regularizes the chiral theory if and only if the theory is free of all perturbative chiral gauge anomalies, including the Lorentz-gauge mixed anomaly. In this explicitly invariant scheme, anomalous theories manifest themselves by having divergent fermion loops

\footnotetext{
${ }^{3}$ It can be shown later on that the regularized gauge current in curved spacetimes does reduce to the original current as the regulator masses go to infinity.

${ }^{4}$ Recall that even a Majorana fermion couples to both the left- and right-handed spin connections.

${ }^{5}$ The representations may be reducible, as in the $\mathrm{SU}(5)$ grand unified theory (GUT) model.
} 
which remain unregularized.

\section{BARE ACTION AND INVARIANT MASSES}

The bare chiral fermion action may be taken to be

$$
\mathcal{S}_{F_{\text {bare }}}=\int d^{4} x e \bar{\Psi}_{L_{0}}^{-} i \not D P_{L} \Psi_{L_{0}}^{-}
$$

where $i \not D=\gamma^{\mu}\left(i \partial_{\mu}+W_{\mu a} T^{a}+\frac{i}{2} A_{\mu A B} \sigma^{A B}\right), \sigma^{A B}=\frac{1}{4}\left[\gamma^{A}, \gamma^{B}\right]$, and $e$ denotes the determinant of the vierbein. $P_{L}=\frac{1}{2}\left(1-\gamma^{5}\right)$ is the left-handed projection operator. We adopt the convention

$$
\left\{\gamma^{A}, \gamma^{B}\right\}=2 \eta^{A B}
$$

with $\eta^{A B}=\operatorname{diag}(-1,+1,+1,+1)$. Lorentz indices are denoted by uppercase Latin indices while Greek indices are spacetime indices.

In general, the fermion multiplet $\Psi_{L_{0}}^{-}$is in a complex representation. Recall that if the generators $T^{a}$ satisfy

$$
\left[T^{a}, T^{b}\right]=i f_{c}^{a b} T^{c},
$$

then $\left(-T^{a}\right)^{*}$ satisfy the same Lie algebra. If If there exists an $S$ such that $S^{-1}\left(-T^{a}\right)^{*} S=T^{a}$, then the representation is called real (pseudoreal) if $S$ is symmetric (antisymmetric). Otherwise, the representation is termed complex.

With only a single left-handed multiplet, Lorentz-invariant mass terms are Majorana in nature. The simple form of $\Psi_{L}^{T} C_{4} \Psi_{L}$ is not invariant under internal symmetry transformations. I] However, with real representations, an invariant mass term $m \Psi_{L}^{T} S C_{4} \Psi_{L}$ can be constructed from a single multiplet. Observe that for a nonvanishing mass term, $S$ has to be symmetric for anticommuting fields and antisymmetric for commuting fields. For complex representations, a gauge and Lorentz invariant mass term cannot be made

\footnotetext{
${ }^{6}$ We adopt the convention of $\left(T^{a}\right)^{\dagger}=T^{a}$ and real structure constants. For invariance of the action, the representation has to be unitary.

${ }^{7} C_{4}$ is the charge conjugation matrix in four dimensions with $C_{4}^{T}=C_{4}^{-1}=C_{4}^{\dagger}=-C_{4}$.
} 
out of a single multiplet. This poses a challenge for the usual invariant PauliVillars-Gupta regularization, even though the chiral fermions may belong to an anomaly-free representation.

We shall generalize the method of Frolov and Slavnov by including regulators which are doubled in internal space. This doubling is achieved by including fermions which transform according to the $\left(-T^{a}\right)^{*}$ representation, and then an invariant mass term can be formed because under

$$
\Psi_{L_{r}}^{-} \rightarrow e^{i \alpha_{a} T^{a}} \Psi_{L_{r}}^{-}, \quad \Psi_{L_{r}}^{+} \rightarrow e^{i \alpha_{a}\left(-T^{a}\right)^{*}} \Psi_{L_{r}}^{+},
$$

the combination $\left[\left(\Psi_{L_{r}}^{+}\right)^{T} C_{4} \Psi_{L_{r}}^{-}+\left(\Psi_{L_{r}}^{-}\right)^{T} C_{4} \Psi_{L_{r}}^{+}+\right.$H.c. $]$is invariant under internal gauge and Lorentz transformations.

We introduce in the enlarged space the quantities

$$
\mathcal{T}^{a} \equiv\left(\begin{array}{cc}
\left(-T^{a}\right)^{*} & 0 \\
0 & T^{a}
\end{array}\right), \quad \sigma^{1} \equiv\left(\begin{array}{cc}
0 & 1_{d} \\
1_{d} & 0
\end{array}\right), \quad \sigma^{3} \equiv\left(\begin{array}{cc}
1_{d} & 0 \\
0 & -1_{d}
\end{array}\right),
$$

where $d$ denotes the number of internal components of the original multiplet. In this notation, the original multiplet can be expressed as

$$
\left[\begin{array}{c}
0 \\
\Psi_{L_{0}}^{-}
\end{array}\right]=\frac{1}{2}\left(1_{2 d}-\sigma^{3}\right) \Psi_{L_{0}}
$$

and the mass terms for the regulator fermions,

$$
\Psi_{L_{r}}=\left[\begin{array}{c}
\Psi_{L_{r}}^{+} \\
\Psi_{L_{r}}^{-}
\end{array}\right]
$$

can be written as $m_{r}\left(\Psi_{L_{r}}^{T} \sigma^{1} C_{4} \Psi_{L_{r}}+\right.$ H.c. $)$. The doubled regulator fermion multiplets are to be coupled to the $2 d$-dimensional representation of the gauge connection, $W_{\mu a} \mathcal{T}^{a}$.

The $\Psi_{L_{r}}$ fields are assumed to be anticommuting. Commuting doubled regulator fields $\Phi_{L_{s}}$ are introduced in a similar manner. These have mass terms

$$
m_{s} \Phi_{L_{s}}^{T}\left(-i \sigma^{2}\right) C_{4} \Phi_{L_{s}}=m_{s}\left[-\left(\Phi_{L_{s}}^{+}\right)^{T} C_{4} \Phi_{L_{s}}^{-}+\left(\Phi_{L_{s}}^{-}\right)^{T} C_{4} \Phi_{L_{s}}^{+}\right]
$$

with

$$
-i \sigma^{2} \equiv\left(\begin{array}{cc}
0 & -1_{d} \\
1_{d} & 0
\end{array}\right)=\sigma^{1} \sigma^{3}
$$


Note that these invariant mass terms for the doubled anticommuting and commuting fields exist, because for the $\mathcal{T}^{a}$ representation, there is a symmetric $\left(\sigma^{1}\right)$ and an antisymmetric $\left(-i \sigma^{2}\right)$ matrix which satisfy

$$
\left(\sigma^{1}\right) \mathcal{T}^{a}\left(\sigma^{1}\right)^{-1}=\left(-i \sigma^{2}\right) \mathcal{T}^{a}\left(-i \sigma^{2}\right)^{-1}=\left(-\mathcal{T}^{a}\right)^{*}
$$

It is clear that these constructs work for arbitrary groups and representations $T^{a}$. Note also that all the fields are left-handed.

We shall next show that this generalization of the Pauli-Villars-Gupta method can regularize chiral fermions perturbatively in the original sense of Frolov and Slavnov if and only if the conditions for perturbative anomaly cancellations, including the mixed Lorentz-gauge anomaly, are satisfied.

\section{REGULARIZED ACTION}

The total regularized action which is explicitly gauge and Lorentz and, also diffeomorphism invariant is taken to be

$$
\begin{aligned}
\mathcal{S}_{F_{r e g}} & =\int d^{4} x e\left[\sum_{r=0,2, \ldots}\left\{\bar{\Psi}_{L_{r}} i \not D \Psi_{L_{r}}+\frac{1}{2} m_{r}\left(\Psi_{L_{r}}^{T} \sigma^{1} C_{4} \Psi_{L_{r}}+\bar{\Psi}_{L_{r}} \sigma^{1} C_{4}^{\dagger} \bar{\Psi}_{L_{r}}^{T}\right)\right\}\right. \\
& \left.-\sum_{s=1,3, \ldots}\left\{\bar{\Phi}_{L_{s}} \sigma^{3} i \not D \Phi_{L_{s}}+\frac{1}{2} m_{s}\left(\Phi_{L_{s}}^{T} \sigma^{1} \sigma^{3} C_{4} \Phi_{L_{s}}+\bar{\Phi}_{L_{s}} C_{4}^{\dagger} \sigma^{3} \sigma^{1} \bar{\Phi}_{L_{s}}^{T}\right)\right\}\right]
\end{aligned}
$$

The sums are over all even natural numbers for the anticommuting fields and over all odd natural numbers for the commuting fields. The usefulness of this convention will become apparent later on. With the exception of

$$
\Psi_{L_{0}}=\frac{1}{2}\left(1-\sigma^{3}\right) \Psi_{L_{0}}=\left[\begin{array}{c}
0 \\
\Psi_{L_{0}}^{-}
\end{array}\right]
$$

\footnotetext{
${ }^{8} \mathrm{~A}$ set of conditions for generalized Pauli-Villars regularization is also given in [7].

${ }^{9}$ We also allow all the fields to transform under general coordinate transformations. Here, we regularize only fermion loops in background fields, and do not address the question of the regularization of the gauge and gravitational fields. Gauge propagators may be regularized by other methods. Full quantum gravity effects are beyond the scope of this paper.
} 
which is the original and undoubled chiral massless $\left(m_{0}=0\right)$ fermion multiplet, all other anticommuting $\Psi_{L_{r}}$ and commuting $\Phi_{L_{s}}$ multiplets are generalized Pauli-Villars-Gupta regulator fields, doubled in internal space, and endowed with Majorana masses, which we take for definiteness to satisfy $m_{n}=n \Lambda$. We emphasize that due to the fact that all the multiplets are lefthanded, there are no couplings to the right-handed spin connection which does not need to be introduced for the Weyl action.

In matrix notation, the regularized fermion action can be reexpressed as

$$
\begin{aligned}
\mathcal{S}_{F_{r e g}} & =\int d x \int d y \frac{1}{2}\left\{\sum_{r}\left[\begin{array}{ll}
\tilde{\Psi}_{L_{r}}^{T}(x) & \tilde{\bar{\Psi}}_{L_{r}}(x)
\end{array}\right]\left[\begin{array}{cc}
M_{r}(x, y) \sigma^{1} C_{4} & -i \not D^{T}(x, y) \\
i \not D(x, y) & M_{r}(x, y) \sigma^{1} C_{4}^{\dagger}
\end{array}\right]\left[\begin{array}{c}
\tilde{\Psi}_{L_{r}}(y) \\
\tilde{\bar{\Psi}}_{L_{r}}^{T}(y)
\end{array}\right]\right. \\
& \left.-\sum_{s}\left[\begin{array}{ll}
\tilde{\Phi}_{L_{s}}^{T}(x) & \tilde{\bar{\Phi}}_{L_{s}}(x)
\end{array}\right]\left[\begin{array}{cc}
M_{s}(x, y) \sigma^{1} \sigma^{3} C_{4} & i \not D^{T}(x, y) \sigma^{3} \\
\sigma^{3} i \not D(x, y) & M_{s}(x, y) C_{4}^{\dagger} \sigma^{3} \sigma^{1}
\end{array}\right]\left[\begin{array}{c}
\tilde{\Phi}_{L_{s}}(y) \\
\tilde{\bar{\Phi}}_{L_{s}}^{T}(y)
\end{array}\right]\right\}, \quad(13)
\end{aligned}
$$

with

$$
\begin{aligned}
M_{n}(x, y) & =m_{n} \delta(x-y) \\
i \not D(x, y) & =e^{\frac{1}{2}}(x) \gamma^{\mu}(x)\left[\left(i \partial_{\mu}^{x}+W_{\mu a}(x) \mathcal{T}^{a}+\frac{i}{2} A_{\mu A B}(x) \sigma^{A B}\right) \delta(x-y)\right] e^{-\frac{1}{2}}(y), \\
i \not D^{T}(x, y) & =e^{-\frac{1}{2}}(x)\left[\left\{i \partial_{\mu}^{y}+W_{\mu a}(y)\left(\mathcal{T}^{a}\right)^{T}+\frac{i}{2} A_{\mu A B}(y)\left(\sigma^{A B}\right)^{T}\right\} \delta(y-x)\right] \gamma^{\mu T}(y) e^{\frac{1}{2}}(y) .
\end{aligned}
$$

To be compatible with the diffeomorphism-invariant measure [17,

$$
\prod_{x, r} D\left[\bar{\Psi}_{L_{r}}(x) e^{\frac{1}{2}}(x)\right] D\left[e^{\frac{1}{2}}(x) \Psi_{L_{r}}(x)\right],
$$

for the fields in curved spacetimes, we have also chosen to use densitized variables defined by

$$
\begin{aligned}
\tilde{\bar{\Psi}}_{L_{r}} & \equiv \bar{\Psi}_{L_{r}} e^{\frac{1}{2}} \\
\tilde{\Psi}_{L_{r}} & \equiv e^{\frac{1}{2}} \Psi_{L_{r}}
\end{aligned}
$$

with a similar set for $\tilde{\bar{\Phi}}_{L_{s}}$ and $\tilde{\Phi}_{L_{s}}$.

For clarity, we shall use the explicit chiral representation

$$
\gamma^{5}=\left(\begin{array}{cc}
1_{2} & 0 \\
0 & -1_{2}
\end{array}\right),
$$




$$
\gamma^{A}=\left(\begin{array}{cc}
0 & i \tau^{A} \\
i \bar{\tau}^{A} & 0
\end{array}\right)
$$

In the above, $\tau^{a}=-\bar{\tau}^{a}(a=1,2,3)$ are Pauli matrices, and $\tau^{0}=\bar{\tau}^{0}=-I_{2}$.

By writing in terms of left-handed two-component Weyl fermions,

$$
\Psi_{L r}=\left[\begin{array}{c}
0 \\
\psi_{r}
\end{array}\right], \quad \Phi_{L s}=\left[\begin{array}{l}
0 \\
\phi_{s}
\end{array}\right]
$$

and $[0$

$$
\left[\begin{array}{ll}
\bar{\psi}_{r} & 0
\end{array}\right] \equiv \bar{\Psi}_{L r}, \quad\left[\bar{\phi}_{s} \quad 0\right] \equiv \bar{\Phi}_{L s}
$$

the propagators in background gauge and gravitational fields can then be read off as

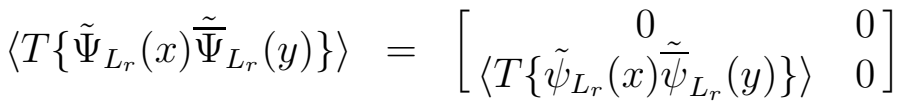

$$
\begin{aligned}
& =-P_{L} e^{-\frac{1}{2}}\left(i D_{\mu}\right)^{\dagger} \gamma^{\mu} e^{\frac{1}{2}} \frac{1}{m_{r}^{2}+e^{\frac{1}{2}} \gamma^{\mu} i D_{\mu} e^{-1}\left(i D_{\nu}\right)^{\dagger} \gamma^{\nu} e^{\frac{1}{2}}} \delta(x-y) \text {. }
\end{aligned}
$$

We have used the identities

$$
\begin{aligned}
\sigma^{1}\left(\mathcal{T}^{a}\right)^{T}\left(\sigma^{1}\right)^{-1} & =-\left(\mathcal{T}^{a}\right)^{\dagger}, \\
C_{4}\left(\sigma^{A B}\right)^{T}\left(C_{4}\right)^{-1} & =-\sigma^{A B} .
\end{aligned}
$$

Furthermore, with respect to the Euclidean inner product $\langle\tilde{X} \mid \tilde{Y}\rangle=\int d^{4} x \tilde{X}^{\dagger}(x) \tilde{Y}(x)$, the Euclidean Dirac operator obeys $\square$

$$
\begin{aligned}
(i \not \mathcal{D})^{\dagger} & \equiv\left(e^{\frac{1}{2}} i \not D e^{-\frac{1}{2}}\right)^{\dagger} \\
& =\left(e^{\frac{1}{2}} \gamma^{\mu} i D_{\mu} e^{-\frac{1}{2}}\right)^{\dagger} \\
& =e^{-\frac{1}{2}}\left(i D_{\mu}\right)^{\dagger} \gamma^{\mu} e^{\frac{1}{2}}
\end{aligned}
$$

\footnotetext{
${ }^{10}$ For Lorentzian signature spacetimes, $\bar{\psi}_{r}=\psi_{r}^{\dagger} \gamma^{0}$ whereas for Euclidean signature, $\bar{\psi}_{r}$ is treated as an independent field.

${ }^{11}$ In writing down the propagator, we do not in general assume the absence of torsion. If the torsion vanishes, then

$$
D_{\mu} e \gamma^{\mu} f=e \gamma^{\mu} D_{\mu} f .
$$


where $^{12}$

$$
\left(i D_{\mu}\right)^{\dagger}=i \partial_{\mu}+W_{\mu a} \mathcal{T}^{{ }^{\dagger}}+\frac{i}{2} A_{\mu A B} \sigma^{A B} .
$$

The Euclidean propagator is therefore

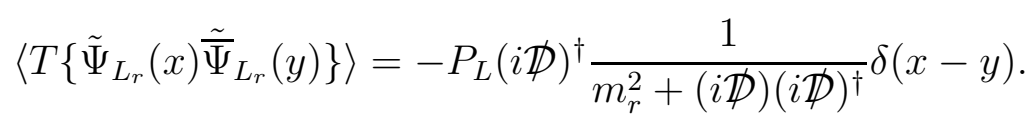

In a similar manner, for the commuting regulators,

$$
\begin{aligned}
\left\langle T\left\{\tilde{\Phi}_{L_{s}}(x) \tilde{\bar{\Phi}}_{L_{s}}(y)\right\}\right\rangle & =\left[\begin{array}{cc}
0 & 0 \\
\left\langle T\left\{\tilde{\phi}_{L_{s}}(x) \tilde{\bar{\phi}}_{L_{s}}(y)\right\}\right\rangle & 0
\end{array}\right] \\
& =P_{L} \sigma^{3}(i \not \mathcal{D})^{\dagger} \frac{1}{m_{s}^{2}+(i \not \mathcal{D})(i \not \mathcal{D})^{\dagger}} \delta(x-y) .
\end{aligned}
$$

\section{REGULARIZED CURRENTS}

The original gauge current is

$$
J^{\mu a}=\frac{\delta \mathcal{S}_{F}}{\delta W_{\mu a}}=\tilde{\bar{\Psi}}_{L_{0}} \gamma^{\mu} \mathcal{T}^{a} \frac{1}{2}\left(1-\sigma^{3}\right) \tilde{\Psi}_{L_{0}} .
$$

With the regulators, the classical current coupled to $W_{\mu a}$ is

$$
\begin{aligned}
J^{\mu a} & =\tilde{\bar{\Psi}}_{L_{0}} \gamma^{\mu} \mathcal{T}^{a} \frac{\left(1-\sigma^{3}\right)}{2} \tilde{\Psi}_{L_{0}}+\sum_{r=2,4, \ldots} \tilde{\bar{\Psi}}_{L_{r}} \gamma^{\mu} \mathcal{T}^{a} \tilde{\Psi}_{L_{r}} \\
& +\sum_{s=1,3, \ldots} \tilde{\bar{\Phi}}_{L_{s}} \gamma^{\mu} \mathcal{T}^{a} \tilde{\Phi}_{L_{s}} .
\end{aligned}
$$

Note that there is a $\frac{1}{2}\left(1-\sigma^{3}\right)$ projection associated with the undoubled original fermion multiplet. As with conventional Pauli-Villars-Gupta regularization, the regulated composite current operator is summarized by 8

$$
\left\langle J^{\mu a}(x)\right\rangle_{r e g}=\lim _{x \rightarrow y} \operatorname{Tr}\left\{-\gamma^{\mu}(x) \mathcal{T}^{a}\left[\frac{1}{2}\left(1-\sigma^{3}\right)\left\langle T\left\{\tilde{\Psi}_{L_{0}}(x) \tilde{\bar{\Psi}}_{L_{0}}(y)\right\}\right\rangle\right.\right.
$$

\footnotetext{
${ }^{12}$ The gauge fields $W_{\mu a}$ and $A_{\mu A B}$ are real and, $\gamma^{A}$ and $i \sigma^{A B}$ are Hermitian for Euclidean signature manifolds.
} 


$$
\left.\left.+\sum_{r=2,4, \ldots}\left\langle T\left\{\tilde{\Psi}_{L_{r}}(x) \tilde{\bar{\Psi}}_{L_{r}}(y)\right\}\right\rangle+\sigma^{3} \sum_{s=1,3, \ldots}\left\langle T\left\{\tilde{\Phi}_{L_{s}}(x) \tilde{\bar{\Phi}}_{L_{s}}(y)\right\}\right\rangle\right]\right\}
$$

The trace runs over Dirac and Yang-Mills indices.

With the expressions for the propagators and the choice of $m_{n}=n \Lambda$ for the regulator masses, we obtain

$$
\begin{aligned}
\left\langle J^{\mu a}(x)\right\rangle_{r e g} & =\lim _{x \rightarrow y} \operatorname{Tr}\left\{\gamma ^ { \mu } ( x ) \mathcal { T } ^ { a } P _ { L } \left[\frac{1}{2}\left(1-\sigma^{3}\right)(i \not \mathcal{D})^{\dagger} \frac{1}{(i \not \mathcal{D})(i \not \mathcal{D})^{\dagger}}+\right.\right. \\
& \left.\left.+\sum_{r=2,4, \ldots}(i \not \mathcal{D})^{\dagger} \frac{1}{r^{2} \Lambda^{2}+(i \not \mathcal{D})(i \not \mathcal{D})^{\dagger}}-\sum_{s=1,3, \ldots}(i \not \mathcal{D})^{\dagger} \frac{1}{s^{2} \Lambda^{2}+(i \not \mathcal{D})(i \not \mathcal{D})^{\dagger}}\right] \delta(x-y)\right\} \\
& =\lim _{x \rightarrow y} \operatorname{Tr}\left\{\gamma^{\mu}(x) \mathcal{T}^{a} \frac{1}{2} P_{L}\left[\frac{1}{i \not \mathcal{D}}\left(\sum_{n=-\infty}^{\infty} \frac{(-1)^{n} \not \mathcal{D} \not{D}^{\dagger}}{n^{2} \Lambda^{2}+\not \mathcal{D} \mathcal{D}^{\dagger}}-\sigma^{3}\right)\right] \delta(x-y)\right\} \\
& \equiv \lim _{x \rightarrow y} \operatorname{Tr}\left\{\gamma^{\mu}(x) \mathcal{T}^{a} \frac{1}{2} P_{L}\left[\frac{1}{i \not \mathcal{D}}\left(f\left(\not \mathcal{D} \mathcal{D}^{\dagger} / \Lambda^{2}\right)-\sigma^{3}\right)\right] \delta(x-y)\right\} .
\end{aligned}
$$

In the above, note that $n$ is summed over all integers.

The effect of the tower of regulators is to replace the divergent bare expression

$$
\left\langle J^{\mu a}\right\rangle_{\text {bare }}=\lim _{x \rightarrow y} \operatorname{Tr}\left\{\gamma^{\mu}(x) \mathcal{T}^{a} P_{L}\left[\frac{1}{i \not D} \frac{1}{2}\left(1-\sigma^{3}\right)\right] \delta(x-y)\right\},
$$

by

$$
\left\langle J^{\mu a}\right\rangle_{\text {reg }}=\lim _{x \rightarrow y} \operatorname{Tr}\left\{\gamma^{\mu}(x) \mathcal{T}^{a} \frac{1}{2} P_{L}\left[\frac{1}{i \not \mathcal{D}}\left(f\left(\not \mathcal{D} \not^{\dagger} / \Lambda^{2}\right)-\sigma^{3}\right)\right] \delta(x-y)\right\} .
$$

This general feature of the effect of the tower shows up in all the regularized gauge currents.

The regulator function

$$
f(z) \equiv \sum_{n=-\infty}^{\infty} \frac{(-1)^{n} z}{n^{2}+z}
$$




$$
=\frac{\pi \sqrt{z}}{\sinh (\pi \sqrt{z})}
$$

has the required properties [1, 07, 8] to ensure convergence. For instance, it falls rapidly to zero as $z \rightarrow \infty$, and when the regulator masses are taken to $\infty, f(0)=1$. However, the $\sigma^{3}$ part of the current remains unmodified, essentially because the tower consists of regulators which are doubled in internal space and is " $\sigma^{3}$ neutral". It, therefore, can regularize only the singlet part of the $\frac{1}{2}\left(1-\sigma^{3}\right)$ projection of the bare current. Thus, it remains to be checked that for chiral theories free of perturbative anomalies, the $\sigma^{3}$ part gives rise to no further divergences, and can in fact be argued to be convergent. When this is true, the total current is then successfully regularized by the tower of regulators.

As an example, to specialize to the original Frolov-Slavnov proposal [1] for $\mathrm{SO}(10)$, we take the $d=16$-dimensional representation of $\operatorname{Spin}(10)$, and set $\Psi_{L}^{-}=\Psi_{16}, \Psi_{L}^{+}=C_{10} \Psi_{\overline{16}}$, and $\sigma^{3}=\Gamma_{11}$. Note that under a gauge transformation,

$\Psi_{16} \rightarrow \exp \left(i \frac{\alpha_{a}}{2} \Sigma^{a}\left(1-\Gamma_{11}\right)\right) \Psi_{16}, \quad C_{10} \Psi_{\overline{16}} \rightarrow \exp \left(-i \frac{\alpha_{a}}{2}\left[\Sigma^{a}\left(1-\Gamma_{11}\right)\right]^{*}\right) C_{10} \Psi_{\overline{16}}$

due to $C_{10} \Sigma^{a} C_{10}^{-1}=-\left(\Sigma^{a}\right)^{T}=-\left(\Sigma^{a}\right)^{*}$ and $\left\{C_{10}, \Gamma_{11}\right\}=0$. Here $\Sigma^{a}$ denote the generators of $\mathrm{SO}(10)$ in the 32 -dimensional representation. Frolov and Slavnov first observed that for the $\mathrm{SO}(10)$ theory with a 16-dimensional Spin(10) chiral multiplet, it can be argued that the $\Gamma_{11}$ part of the current gives rise to no divergences in four dimensions since by power counting, the amplitudes for the relevant divergent fermion loop diagrams are proportional to the traces of four or fewer generators of $\operatorname{Spin}(10)$ with $\Gamma_{11}$. These traces vanish identically. For arbitrary anomaly-free representations, we shall next argue in the same spirit that the analogous $\sigma^{3}$ part of the currents gives rise to no further divergences. 


\section{ANOMALIES AND CONDITIONS FOR REGULARIZATION}

We shall expand the vierbein as $E_{A}^{\mu}=\delta_{A}^{\mu}+h_{A}^{\mu}$ and the rest of the fields about zero. Then

$$
\begin{aligned}
i \not D & =e^{\frac{1}{2}} i \not D e^{-\frac{1}{2}} \\
& \equiv i \not \partial_{f}+\not B
\end{aligned}
$$

where

$$
i \partial_{f}=i \gamma^{A} \delta_{A}^{\mu} \partial_{\mu}
$$

is the flat spacetime Dirac operator in the absence of all gauge fields, and

$$
\begin{aligned}
\not B & =\gamma^{A}\left[h_{A}^{\mu} i \partial_{\mu}+\left(\delta_{A}^{\mu}+h_{A}^{\mu}\right)\left(\frac{i}{2} A_{\mu A B} \sigma^{A B}-\frac{i}{2} \Gamma_{\nu \mu}^{\nu}+W_{\mu a} \mathcal{T}^{a}\right)\right], \\
\Gamma_{\nu \mu}^{\nu} & \equiv \partial_{\mu}(\ln e) .
\end{aligned}
$$

The inverse operator has the expansion

$$
\frac{1}{i \not \supset}=\frac{1}{i \not \partial_{f}}+\frac{1}{i \not \partial_{f}}(-\not B) \frac{1}{i \not \partial}_{f}+\frac{1}{i \not \partial_{f}}(-\not B) \frac{1}{i \not \partial_{f}}(-\not B) \frac{1}{i \not \partial}_{f}+\ldots
$$

which can be substituted into expression (32). Fermion loops or perturbative multipoint correlation functions can be generated by functionally differentiating the regularized currents with respect to the fields.

In order to obtain the conditions that guarantee no divergences from the unregulated $\sigma^{3}$ part of (32), we may note that $\operatorname{Tr}\left(\sigma^{3}\right)=0$ and $\not B$ is linear in $\mathcal{T}^{a}$. The only term containing $\mathcal{T}^{a}$ is $E_{A}^{\mu} W_{\mu a} \mathcal{T}^{a}$. Furthermore, nonvanishing diagrams from the unregulated $\sigma^{3}$ part must involve

$$
\operatorname{Tr}\left\{\sigma^{3} \mathcal{T}^{a_{1}} \ldots \mathcal{T}^{a_{n}}\right\} \neq 0
$$

for some value of $n$. Here, the trace is over internal indices.

To obtain the condition for convergence, we can consider a generic fermion loop diagram with nonvanishing amplitude involving this condition. The 
vertices can be separated into those which involve $\mathcal{T}^{a}$ and those which do not. Let $n$ and $k$ be the number of these vertices respectively. Potential complications due to curved spacetimes come essentially from the $h_{A}^{\mu}$ terms. Contributions from the Lorentz connection are rather straightforward since the coupling involves no derivatives and there is also no coupling between $A_{\mu A B}$ and $\mathcal{T}^{a}$. Diagrams involving vertices due to the $\bar{\Psi}_{L} h_{A}^{\mu} i \gamma^{A} \partial_{\mu} \Psi_{L}$ terms have derivative couplings. These vertices carry weight $\gamma^{A} i \partial_{\mu}$ and diverge linearly. Thus, the most divergent fermion loop diagrams obeying Eq. (39) involve, in general, up to $k$ of these $i \gamma^{A} \partial_{\mu}$ vertices, $m$ vertices due to couplings of the type $\bar{\Psi}_{L} h_{A}^{\mu} i \gamma^{A} W_{\mu a} \mathcal{T}^{a} \Psi_{L}$, and $(n-m)$ vertices from $\bar{\Psi}_{L} i \gamma^{A} \delta_{A}^{\mu} W_{\mu a} \mathcal{T}^{a} \Psi_{L}$ couplings. ${ }^{13}$ Note also that each propagator between vertices costs $\not_{f}{ }^{-1}$. For the purpose of power counting such fermion loops, the amplitude therefore behaves symbolically like

$$
\sim \operatorname{Tr}\left[\sigma^{3} \mathcal{T}^{a_{1}} \ldots \mathcal{T}^{a_{n}}\right]\left(\partial^{k} \frac{1}{\not \partial_{f}^{m+(n-m)+k}}\right) .
$$

In momentum space in four dimensions, on integrating over the loop momentum, this goes like

$$
\sim \int d^{4} p \frac{1}{p^{n}} \operatorname{Tr}\left\{\sigma^{3} \mathcal{T}^{a_{1}} \ldots \mathcal{T}^{a_{n}}\right\}
$$

Clearly, the degree of divergence is $(4-n)$. Therefore, if we demand that

$$
\operatorname{Tr}\left\{\sigma^{3} \mathcal{T}^{a_{1}} \ldots \mathcal{T}^{a_{n}}\right\}=0 \quad \text { for } n \leq 4,
$$

we can argue, as Frolov and Slavnov did for $\mathrm{SO}(10)$, that the $\sigma^{3}$ part of the current gives rise to no further divergences, and the whole expression (32) is indeed regularized by the tower of regulators. The condition (42) also suggests generalizations to spacetime dimensions other than four. In terms of the $T^{a}$ representation of the original multiplet, Eq. (42) translates into

$$
\operatorname{Tr}\left\{T^{a_{1}} \ldots T^{a_{n}}\right\}=\operatorname{Tr}\left\{\left(-T^{a_{1}}\right)^{*} \ldots\left(-T^{a_{n}}\right)^{*}\right\}, \quad n \leq 4 .
$$

For real and pseudodreal representations, where $\left(-T^{a}\right)^{*}=S T^{a} S^{-1}$, the condition is obviously satisfied. This means that all such representations

\footnotetext{
13 The number of the two type of vertices involving $\mathcal{T}^{a}$ must sum up to $n$ to be compatible with Eq. (39).
} 
are free of perturbative gauge anomalies since the proposed regularization which explicitly preserves the gauge symmetries works. Although it is true that with a different set of regulator masses it may be possible to regularize a theory with real representation by a finite tower instead of the infinite one we have presented, we nevertheless would like to discuss the issue in a more general context. In this fashion, we will obtain a general condition and regularization scheme for arbitrary representations including both complex and real and pseudoreal representations[0]. However, it may be worthwhile to note that for real and pseudoreal representations, the condition

$$
\operatorname{Tr}\left\{\sigma^{3} \mathcal{T}^{a_{1}} \ldots \mathcal{T}^{a_{n}}\right\}=0
$$

is satisfied for all values of $n$. Thus the $\sigma^{3}$ contribution of the current can be argued to be absent even for convergent diagrams. Therefore one can argue that the regularized current is

$$
\left\langle J^{\mu a}\right\rangle_{\text {reg }}=\lim _{x \rightarrow y} \operatorname{Tr}\left\{\gamma^{\mu}(x) \mathcal{T}^{a} \frac{1}{2} P_{L} \frac{1}{i \not \mathcal{D}} f\left(\not \mathcal{D}^{\dagger} / \Lambda^{2}\right) \delta(x-y)\right\} .
$$

A more careful consideration with different choices of the regulator masses shows that it is in fact possible to write the regularized currents for real and pseudoreal representations in the above form with the appropriate regulator functions $[7$.

We shall now present the complete solution for arbitrary representations including complex ones, and show that the invariant regularization scheme works if and only if the chiral theory is free of all perturbative gauge anomalies, including the mixed Lorentz-gauge anomaly.

To begin, note that since the generators are Hermitian, $\left(T^{a}\right)^{*}=\left(T^{a}\right)^{T}$, and condition (43) is the same as

$$
\begin{aligned}
\operatorname{Tr}\left\{T^{a_{1}} \ldots T^{a_{n}}\right\} & =(-1)^{n} \operatorname{Tr}\left\{\left(T^{a_{1}}\right)^{T} \ldots\left(T^{a_{n}}\right)^{T}\right\} \\
& =(-1)^{n} \operatorname{Tr}\left\{\left(T^{a_{n}}\right) \ldots\left(T^{a_{1}}\right)\right\}, \quad n \leq 4 .
\end{aligned}
$$

For $n=2$, the equation is trivially statisfied due to the cyclic property of the trace, and this imposes no constraints on $T^{a}$. The cases $n=1$ and $n=3$ translate precisely into

$$
\operatorname{Tr}\left(T^{a}\right)=0
$$


and

$$
\operatorname{Tr}\left(T^{a}\left\{T^{b}, T^{c}\right\}\right)=0
$$

respectively.

The case of $n=4$ imposes no new restrictions if the condition for $n=3$ is satisfied. To see this, we decompose the $n=4$ condition into antisymmetric and symmetric parts by writing

$$
\begin{aligned}
\operatorname{Tr}\left(\sigma^{3} \mathcal{T}^{a} \mathcal{T}^{b} \mathcal{T}^{c} \mathcal{T}^{d}\right) & =\frac{1}{2} \operatorname{Tr}\left(\sigma^{3}\left[\mathcal{T}^{a}, \mathcal{T}^{b}\right] \mathcal{T}^{c} \mathcal{T}^{d}\right)+\frac{1}{2} \operatorname{Tr}\left(\sigma^{3}\left\{\mathcal{T}^{a}, \mathcal{T}^{b}\right\} \mathcal{T}^{c} \mathcal{T}^{d}\right) \\
& =\frac{i}{2} f^{a b}{ }_{e} \operatorname{Tr}\left(\sigma^{3} \mathcal{T}^{e} \mathcal{T}^{c} \mathcal{T}^{d}\right)+\frac{1}{4} \operatorname{Tr}\left(\sigma^{3}\left\{\mathcal{T}^{a}, \mathcal{T}^{b}\right\}\left[\mathcal{T}^{c}, \mathcal{T}^{d}\right]\right) \\
& +\frac{1}{4} \operatorname{Tr}\left(\sigma^{3}\left\{\mathcal{T}^{a}, \mathcal{T}^{b}\right\}\left\{\mathcal{T}^{c}, \mathcal{T}^{d}\right\}\right)
\end{aligned}
$$

The first two of these terms vanish due to $\left[\mathcal{T}^{a}, \mathcal{T}^{b}\right]=i$ f ${ }^{a b}{ }_{c} \mathcal{T}^{c}$, and the $n=3$ condition

$$
\operatorname{Tr}\left(\sigma^{3} \mathcal{T}^{a} \mathcal{T}^{b} \mathcal{T}^{c}\right)=0
$$

The final term also vanishes, since, in terms of $T^{a}$,

$$
\begin{aligned}
\operatorname{Tr}\left(\sigma^{3}\left\{\mathcal{T}^{a}, \mathcal{T}^{b}\right\}\left\{\mathcal{T}^{c}, \mathcal{T}^{d}\right\}\right) & =\operatorname{Tr}\left(\left\{T^{a}, T^{b}\right\}\left\{T^{c}, T^{d}\right\}\right)-\operatorname{Tr}\left(\left\{\left(T^{a}\right)^{*},\left(T^{b}\right)^{*}\right\}\left\{\left(T^{c}\right)^{*},\left(T^{d}\right)^{*}\right\}\right) \\
& =\operatorname{Tr}\left(\left\{T^{a}, T^{b}\right\}\left\{T^{c}, T^{d}\right\}\right)-\operatorname{Tr}\left(\left\{\left(T^{a}\right)^{T},\left(T^{b}\right)^{T}\right\}\left\{\left(T^{c}\right)^{T},\left(T^{d}\right)^{T}\right\}\right) \\
& =\operatorname{Tr}\left(\left\{T^{a}, T^{b}\right\}\left\{T^{c}, T^{d}\right\}\right)-\operatorname{Tr}\left(\left\{T^{c}, T^{d}\right\}\left\{T^{a}, T^{b}\right\}\right)^{T} \\
& =0 .
\end{aligned}
$$

Thus the constraints on $T^{a}$ come only from the $n=1$ and $n=3$ restrictions. The first traceless constraint is precisely the condition in four dimensions for the cancellation of the mixed Lorentz-gauge anomaly 11, while the second is the requirement for the cancellation of perturbative chiral gauge anomalies [18]. Therefore, we can conclude that the success of this invariant scheme is synonymous with the absence of all perturbative gauge anomalies. In this scheme, it is clear that anomalous chiral theories manifest themselves by having unregularized divergent fermion loops, whereas anomaly-free theories are regularized in an explicitly invariant manner. 


\section{GRAVITATIONAL CURRENTS}

We emphasize that in this proposed regularization scheme, all the fermions are left-handed, and these are coupled to only the left handed projection of the spin connection rather than the full spin connection. In the chiral representation,

$$
\frac{i}{2} A_{\mu A B} \sigma^{A B} P_{L}=\left[\begin{array}{cc}
0 & 0 \\
0 & A_{\mu a}^{-} \frac{\tau^{a}}{2}
\end{array}\right]
$$

Note that

$$
\begin{aligned}
A_{\mu a}^{-} \frac{\tau^{a}}{2} & =\left[i A_{\mu 0 a}-\frac{1}{2} \epsilon_{a}^{b c} A_{\mu b c}\right] \frac{\tau^{a}}{2} \\
& =-\frac{i}{4} A_{\mu A B} \bar{\tau}^{A} \tau^{B}
\end{aligned}
$$

is also precisely the Ashtekar connection in the (anti-)self-dual formulation of gravity in four dimensions [13, 14]. In this context, only left-handed fermions are allowed[15, 16]. Thus the regularization scheme studied here is well suited for the description of all the four known forces in a completely chiral fashion[16]. In what follows, we will suppose, as in the first order formulation, that the spin connection and the vierbein are independent, and that the torsion is not necessarily zero.

We can compute the the current coupled to the spin connection, $A_{\mu A B}$, by using the method of the previous sections. In effect, the currents are obtained by the replacement of $\mathcal{T}^{a}$ by $\frac{i}{2} \sigma^{A B}$ in expressions (28) to (30). The results are

$$
J^{\mu A B}=\tilde{\bar{\Psi}}_{L_{0}} \gamma^{\mu} \frac{i}{2} \sigma^{A B} P_{L} \frac{1}{2}\left(1-\sigma^{3}\right) \tilde{\Psi}_{L_{0}} .
$$

and

$$
\begin{aligned}
\left\langle J^{\mu A B}(x)\right\rangle_{r e g} & =\lim _{x \rightarrow y} \operatorname{Tr}\left\{\gamma ^ { \mu } ( x ) \frac { i } { 2 } \sigma ^ { A B } P _ { L } \left[\frac{1}{2}\left(1-\sigma^{3}\right)(i \not \mathcal{D})^{\dagger} \frac{1}{(i \not \mathcal{D})(i \not \mathcal{D})^{\dagger}}\right.\right. \\
& \left.\left.+\sum_{r=2,4, \ldots}(i \not \mathcal{D})^{\dagger} \frac{1}{r^{2} \Lambda^{2}+(i \not \mathcal{D})(i \not \mathcal{D})^{\dagger}}-\sum_{s=1,3, \ldots}(i \not \mathcal{D})^{\dagger} \frac{1}{s^{2} \Lambda^{2}+(i \not \mathcal{D})(i \not \mathcal{D})^{\dagger}}\right] \delta(x-y)\right\} \\
& =\lim _{x \rightarrow y} \operatorname{Tr}\left\{\gamma^{\mu}(x) \frac{i}{2} \sigma^{A B} \frac{1}{2} P_{L}\left[\frac{1}{i \not \mathcal{D}}\left(f\left(\not \mathcal{D} \not^{\dagger} / \Lambda^{2}\right)-\sigma^{3}\right)\right] \delta(x-y)\right\} .
\end{aligned}
$$


Again, the presence of the tower of regulators serves to replace the divergent bare expression

$$
\left\langle J^{\mu A B}\right\rangle_{\text {bare }}=\lim _{x \rightarrow y} \operatorname{Tr}\left\{\gamma^{\mu}(x) \frac{i}{2} \sigma^{A B} P_{L}\left[\frac{1}{i \not D} \frac{1}{2}\left(1-\sigma^{3}\right)\right] \delta(x-y)\right\}
$$

by

$$
\left\langle J^{\mu A B}\right\rangle_{r e g}=\lim _{x \rightarrow y} \operatorname{Tr}\left\{\gamma^{\mu}(x) \frac{i}{2} \sigma^{A B} \frac{1}{2} P_{L}\left[\frac{1}{i \not D}\left(f\left(\not \mathcal{D}^{\dagger} / \Lambda^{2}\right)-\sigma^{3}\right)\right] \delta(x-y)\right\} .
$$

The arguments of the last section with regard to the unregularized $\sigma^{3}$ part can be repeated, and we conclude that Eqs. (47) and (48) are again the precise conditions for the current to be free of divergences.

Next, we discuss the energy-momentum tensor. Various proposals for defining the energy momentum tensor have been suggested [17]. If the classical bare action is regarded as $S_{F}\left(\bar{\Psi}_{L_{0}}, \Psi_{L_{0}}, E_{A}^{\mu}, W_{\mu a}, A_{\mu A B}\right)$, then the energy momentum tensor $\Theta_{\mu \nu}$ is obtained from

$$
\begin{aligned}
e \Theta_{\mu \nu} & =e_{\mu A} \frac{\delta S_{F}}{\delta E_{A}^{\nu}} \\
& =\bar{\Psi}_{L_{0}} \gamma_{\mu} i D_{\nu} \Psi_{L_{0}}-g_{\mu \nu} \mathcal{L}
\end{aligned}
$$

where $\mathcal{L}$ is the Lagrangian. On the other hand, if the variables $\tilde{\bar{\Psi}}_{L}$ and $\tilde{\Psi}_{L}$ are to be treated as independent integration variables as is suggested by the diffeomorphism-invariant measure (15), then the energy momentum tensor $T_{\mu \nu}$ regarded as the source current for the background vierbein is

$$
e T_{\mu \nu}=e_{\mu A} \frac{\delta \tilde{S}_{F}}{\delta E_{A}^{\nu}}
$$

with

$$
\begin{aligned}
\tilde{S}_{F}\left(\tilde{\bar{\Psi}}_{L_{0}}, \tilde{\Psi}_{L_{0}}, E_{A}^{\mu}, W_{\mu a}, A_{\mu A B}\right) & =\int d^{4} x \tilde{\bar{\Psi}}_{L_{0}} e^{\frac{1}{2}} i \not D e^{-\frac{1}{2}} \tilde{\Psi}_{L_{0}} \\
& =\int d^{4} x \tilde{\bar{\Psi}}_{L_{0}} E_{A}^{\mu} \gamma^{A}\left[i D_{\mu}-\frac{i}{2}\left(\partial_{\mu} \ln e\right)\right] \tilde{\Psi}_{L_{0}}
\end{aligned}
$$


The expression for the corresponding energy-momentum tensor is then

$$
e T_{\mu \nu}=\tilde{\bar{\Psi}}_{L_{0}} \gamma_{\mu} i\left(D_{\nu}-\frac{1}{2} \Gamma_{\alpha \nu}^{\alpha}\right) \tilde{\Psi}_{L_{0}}-\frac{i}{2} g_{\mu \nu} \partial_{\alpha}\left(\tilde{\bar{\Psi}}_{L_{0}} \gamma^{\alpha} \tilde{\Psi}_{L_{0}}\right)
$$

In terms of variables which are not densitized,

$$
\left.T_{\mu \nu}=\bar{\Psi}_{L_{0}} \gamma_{\mu} i D_{\nu} \Psi_{L_{0}}-\frac{i}{2} g_{\mu \nu}\left[\partial_{\alpha}\left(\bar{\Psi}_{L_{0}} \gamma^{\alpha} \Psi_{L_{0}}\right)+\Gamma_{\beta \alpha}^{\beta} \bar{\Psi}_{L_{0}} \gamma^{\alpha} \Psi_{L_{0}}\right)\right]
$$

As a result, $T_{\mu \nu}$ and $\Theta_{\mu \nu}$ are related by

$$
e T_{\mu \nu}=e \Theta_{\mu \nu}+\frac{1}{2} g_{\mu \nu}\left(\tilde{\bar{\Psi}}_{L_{0}} \frac{\delta \tilde{S}_{F}}{\delta \tilde{\Psi}_{L_{0}}}+\tilde{\Psi}_{L_{0}} \frac{\delta \tilde{S}_{F}}{\delta \tilde{\Psi}_{L_{0}}}\right) .
$$

The difference between the two is therefore not significant classically when the equations of motion can be imposed. However, at the quantum level, there can be subtleties[17]. Because of the choice of the densitized variables, all bare mass terms and, in particular, regulator mass terms, are independent of the vierbein and therefore do not contribute to $T_{\mu \nu}$. The total energymomentum tensor will include the kinetic, but no mass, contributions of all the anticommuting and commuting regulators. The regularized expression becomes

$$
\begin{aligned}
\left\langle e T_{\mu \nu}\right\rangle_{r e g} & =\lim _{x \rightarrow y} \operatorname{Tr}\left\{\gamma_{\mu} i\left(D_{\nu}-\frac{1}{2} \Gamma_{\alpha \nu}^{\alpha}\right) \frac{1}{i \not D} P_{L} \frac{1}{2}\left[f\left(\frac{\not D D^{\dagger}}{\Lambda^{2}}\right)-\sigma^{3}\right] \delta(x-y)\right\} \\
& +\frac{i}{2} g_{\mu \nu}\left\langle\partial_{\alpha} J_{5}^{\alpha}\right\rangle
\end{aligned}
$$

where $J_{5}^{\alpha}$ is the ABJ current which will be discussed more fully in the next section:

$$
J_{5}^{\mu}=-\sum_{r=0,2, \ldots} \tilde{\bar{\Psi}}_{L_{r}} \gamma^{\mu} \tilde{\Psi}_{L_{r}}+\sum_{s=1,3, \ldots} \tilde{\bar{\Phi}}_{L_{s}} \sigma^{3} \gamma^{\mu} \tilde{\Phi}_{L_{s}} .
$$

Again, a slight variation of the previous arguments with regard to relevant diagrams proves that the $\sigma^{3}$ part of the energy-momentum tensor gives rise to no divergent fermion loops if conditions (47) and (48) hold. Hence the expression for the energy-momentum tensor is regularized for finite $\Lambda$.

In our present discussion, we do not densitize the background variables and eschew use, for instance, of $W_{A a} \equiv e^{\frac{1}{2}} E_{A}^{\mu} W_{\mu a}$ instead of $W_{\mu a}$. This choice 
would be useful if an explicitly diffeomorphism invariant measure $\prod D W_{A a}$ is required when the path integral formalism is to be applied to the quantization of the gauge fields [17]. In this paper, gauge and gravitational fields are to be treated as background fields only.

The energy-momentum tensor should be symmetrized if it is to be regarded as the source of the metric. It is known that there are no perturbative Lorentz anomalies in four dimensions [19]. This is verified by the explicitly Lorentz-invariant regularization scheme proposed here. If the vierbein and the left-handed spin connection are to be dynamically described by the (anti)self-dual formulation of gravity [13, 14, then the energy momentum tensor appears as the source on the right hand side of the corresponding equation of motion. We also do not Hermitize the Weyl action. The difference between the Weyl action and the Hermitian version involves the divergence of the ABJ current and also torsion terms and, is given by Eq. (36) of Ref. [16. As a result, among other things, the energy-momentum tensor presented here picks up an imaginary term (in Lorentzian signature spacetimes) proportional to the divergence of the chiral current. Since the expectation value of the divergence of the ABJ current is not zero quantum mechanically, there can be subtle violations of discrete symmetries due to the ABJ anomaly[20], especially in the presence of topologically nontrivial gauge and gravitational instantons, and also nonvanishing torsion. Details of consequences of these violations will be presented elsewhere.

\section{VII. $\gamma^{5}$ ANOMALY}

The regularization of gauge singlet currents requires a separate discussion. The ABJ current has aleady appeared above in the regularized expression for the energy-momentum tensor Eq. (64) and, as we shall see, plays a critical role in constraining the fermion content of the theory [16].

Under a singlet chiral $\gamma^{5}$ rotation,

$$
\begin{aligned}
\tilde{\Psi}_{L_{r}} \rightarrow e^{i \alpha \gamma^{5}} \tilde{\Psi}_{L_{r}} & =e^{-i \alpha} \tilde{\Psi}_{L_{r}}, \\
\tilde{\bar{\Psi}}_{L_{r}} \rightarrow \tilde{\bar{\Psi}}_{L_{r}} e^{i \alpha \gamma^{5}} & =\tilde{\bar{\Psi}}_{L_{r}} e^{i \alpha},
\end{aligned}
$$


and similarly for $\tilde{\Phi}_{L_{s}}$ and $\tilde{\bar{\Phi}}_{L_{s}}$. Kinetic terms are invariant under this global tranformation, but mass terms are not. The bare massless action is invariant under such a global transformation, and the associated ABJ or $\gamma^{5}$ current

$$
J_{5}^{\mu}=\tilde{\bar{\Psi}}_{L_{0}} \gamma^{\mu} \gamma^{5} \tilde{\Psi}_{L_{0}}=-\tilde{\bar{\Psi}}_{L_{0}} \gamma^{\mu} \tilde{\Psi}_{L_{0}}=-J_{F}^{\mu}
$$

is conserved classically, i.e.. $\partial_{\mu} J_{5}^{\mu}=0$. However, the bare quantum composite current

$$
\left\langle J_{5}^{\mu}\right\rangle_{\text {bare }}=-\lim _{x \rightarrow y} \operatorname{Tr}\left\{\gamma^{\mu}(x) P_{L}\left[\frac{1}{i \not D} \frac{1}{2}\left(1-\sigma^{3}\right)\right] \delta(x-y)\right\}
$$

is divergent. The regularized current is not necessarily conserved. In the generalized Pauli-Villars-Gupta scheme, the mass terms of the regulators break the symmetry explicitly. So for the ABJ current, even at the classical level, the current including the regulators is only partially conserved. The relation is

$$
\begin{aligned}
\partial_{\mu} J_{5}^{\mu}= & \quad i\left[\sum_{r=2,4, \ldots} m_{r}\left(\tilde{\Psi}_{L_{r}}^{T} \sigma^{1} C_{4} \tilde{\Psi}_{L_{r}}-\tilde{\bar{\Psi}}_{L_{r}} C_{4}^{\dagger} \sigma^{1} \tilde{\bar{\Psi}}_{L_{r}}^{T}\right)\right. \\
& \left.-\sum_{s=1,3, \ldots} m_{s}\left(\tilde{\Phi}_{L_{s}}^{T} \sigma^{1} \sigma^{3} C_{4} \tilde{\Phi}_{L_{s}}-\tilde{\bar{\Phi}}_{L_{s}} C_{4}^{\dagger} \sigma^{3} \sigma^{1} \tilde{\bar{\Phi}}_{L_{s}}^{T}\right)\right]
\end{aligned}
$$

with

$$
J_{5}^{\mu}=-\sum_{r=0,2, \ldots} \tilde{\bar{\Psi}}_{L_{r}} \gamma^{\mu} \tilde{\Psi}_{L_{r}}+\sum_{s=1,3, \ldots} \tilde{\bar{\Phi}}_{L_{s}} \sigma^{3} \gamma^{\mu} \tilde{\Phi}_{L_{s}} .
$$

The expectation value of the regularized ABJ current is

$$
\begin{aligned}
\left\langle J_{5}^{\mu}(x)\right\rangle_{r e g} & =-\lim _{x \rightarrow y} \operatorname{Tr}\left\{\gamma ^ { \mu } ( x ) \frac { i } { 2 } P _ { L } \left[\frac{1}{2}\left(1-\sigma^{3}\right)(i \not \mathcal{D})^{\dagger} \frac{1}{(i \not \mathcal{D})(i \not \mathcal{D})^{\dagger}}\right.\right. \\
& \left.\left.+\sum_{r=2,4, \ldots}(i \not \mathcal{D})^{\dagger} \frac{1}{r^{2} \Lambda^{2}+(i \not \mathcal{D})(i \not \mathcal{D})^{\dagger}}-\sum_{s=1,3, \ldots}(i \not \mathcal{D})^{\dagger} \frac{1}{s^{2} \Lambda^{2}+(i \not \mathcal{D})(i \not \mathcal{D})^{\dagger}}\right] \delta(x-y)\right\} \\
& =-\lim _{x \rightarrow y} \operatorname{Tr}\left\{\gamma^{\mu}(x) \frac{1}{2}\left(1-\gamma^{5}\right) \frac{1}{i \not \mathcal{D}} \frac{1}{2}\left(f\left(\mathcal{D D D}^{\dagger} / \Lambda^{2}\right)-\sigma^{3}\right) \delta(x-y)\right\} .
\end{aligned}
$$


The previous arguments concerning the unregulated $\sigma^{3}$ part are still valid. Within this context, we have in effect regularized the ABJ current, and the associated amplitudes can be computed explicitly.

There is nevertheless a subtlety which we have glossed over. The transformation given by Eq. (66) rotates fields by the same phase, independently of their quantum numbers under the gauge group. That is the reason why mass terms are not left invariant. We could suppose, on the other hand, that the phase transformation on $\tilde{\Psi}_{L_{0}}$ is generated by fermion number and consider the regulator fields to consist of fermions and antifermions. In this fashion, the relevant fermion number current can be written as

$$
J_{f}^{\mu}=\sum_{r=0,2, \ldots} \tilde{\bar{\Psi}}_{L_{r}} \sigma^{3} \gamma^{\mu} \tilde{\Psi}_{L_{r}}+\sum_{s=1,3, \ldots} \tilde{\bar{\Phi}}_{L_{s}} \gamma^{\mu} \tilde{\Phi}_{L_{s}}
$$

This current should be conserved classically. However, a straightforward repeat of the arguments above now shows that it is not regularized. The factor of $\left(1-\sigma^{3}\right)$ that appears in the primary field $\tilde{\Psi}_{L_{0}}$ is modified upon regularization to $\left(1-f \sigma^{3}\right)$. As a result, regularization affects the part of the amplitude which was convergent because of Eqs. (47) and (48), but leaves the remainder divergent.

It is interesting to note that a similar phenomenon takes place in the vectorlike formulation 8]. If we double in external rather than internal space by including bispinors regulators, then the corresponding result for the fermion current is

$$
\left\langle J_{f}^{\mu}\right\rangle_{\text {reg }}=-\lim _{x \rightarrow y} \operatorname{Tr}\left\{\gamma^{\mu}(x) \frac{1}{2}\left(f\left(\not \mathcal{D} \mathbb{D}^{\dagger} / \Lambda^{2}\right)-\gamma^{5}\right) \frac{1}{i \not \mathcal{D}} \frac{1}{2}\left(1-\sigma^{3}\right) \delta(x-y)\right\} .
$$

In this scheme, ${ }^{\text {th }}$ potentially unregularized divergences can come from the $\gamma^{5}\left(1-\sigma^{3}\right)$ part of $J_{f}^{\mu}$. For gauge currents, parity-odd divergent contributions from fermion loops cancel for anomaly-free gauge theories. However, for $J_{f}^{\mu}$, parity-odd amplitudes from triangle diagrams remain unaffected by these restrictions and are divergent [8]. For the axial current, the regularized chiral projection operator appears as $\left(1-f \gamma_{5}\right)$, and there are potential divergences in the parity-conserving part of the amplitude. Nonetheless, it can be shown

\footnotetext{
${ }^{14}$ In the vectorlike formulation, the doubling is in external space, and the covariant derivative in Eq. (73) contains $W_{\mu a} T^{a}$ rather than $W_{\mu a} \mathcal{T}^{a}$.
} 
that the divergent diagrams cancel when the anomaly cancellation conditions hold [7].

In the chiral scheme, it is possible to define the fermion current via the ABJ current, $J_{F}^{\mu}=-J_{5}^{\mu}$, with both currents carrying weight 1 as a result of the choice of the densitized commuting and anticommuting variables. This identification is consistent with the original degeneracy present in the bare action. Note that the chiral regularization preserves this degeneracy ${ }^{15}$. No such definition of regularized singlet currents free of divergences is possible within the vectorlike scheme without further auxilliary regularization prescriptions[8].

The ABJ anomaly can be explicitly computed by taking the divergence of the expectation value of the regularized expression (71). Here we choose to compute the explicit divergence of the chiral current as

$$
\left\langle\partial_{\mu} J_{5}^{\mu}\right\rangle_{r e g}=\partial_{\mu} \lim _{x \rightarrow y} \operatorname{Tr}\left\{-\gamma^{\mu} \frac{1}{2}\left(1-\gamma^{5}\right) \frac{1}{i \not D} \frac{1}{2}\left(f\left(\mathcal{D}^{\dagger}{ }^{\dagger} / \Lambda^{2}\right)-\sigma^{3}\right) \delta(x-y)\right\} .
$$

The trace can be evaluated by using the complete sets of eigenvectors, $\left\{X_{n}\right\}$ and $\left\{Y_{n}\right\}$, of the positive-semidefinite Hermitian operators with

$$
\begin{aligned}
\not \mathcal{D} \mathcal{D}^{\dagger} X_{n} & =\lambda_{n}^{2} X_{n}, \\
\mathcal{D}^{\dagger} \not \mathcal{D} Y_{n} & =\lambda_{n}^{2} Y_{n} .
\end{aligned}
$$

For the modes with nonzero eigenvalues, $X_{n}$ and $Y_{n}$ are paired by

$$
X_{n}=\not \mathcal{D} Y_{n} / \lambda_{n}, \quad Y_{n}=\not{D}^{\dagger} X_{n} / \lambda_{n}
$$

Consequently, this yields

$$
\begin{aligned}
\left\langle\partial_{\mu} J_{5}^{\mu}\right\rangle_{r e g} & =-\partial_{\mu}\left[\sum_{n} X_{n}^{\dagger} \gamma^{\mu} P_{L}(i \not \mathcal{D})^{\dagger} \frac{1}{\left.\mathcal{D}^{\dagger}\right)^{\dagger}} \frac{1}{2}\left(f\left(\not \mathcal{D} \mathcal{D}^{\dagger} / \Lambda^{2}\right)-\sigma^{3}\right) X_{n}\right] \\
& =i \partial_{\mu}\left[\sum_{n} X_{n}^{\dagger} \gamma^{\mu} P_{L} \frac{1}{2 \lambda_{n}}\left(f\left(\lambda_{n}^{2} / \Lambda^{2}\right)-\sigma^{3}\right) Y_{n}\right]
\end{aligned}
$$

\footnotetext{
${ }^{15}$ See also Eqs. (66) and (67).

${ }^{16} \mathrm{It}$ is assumed that zero modes have been subtracted from the expectation value of the current. They do not occur in the action in the path integral formulation 21.
} 


$$
\begin{aligned}
& =i\left[\sum_{n} \partial_{\mu}\left(X_{n}^{\dagger} \gamma^{\mu}\right) P_{L} \frac{1}{2 \lambda_{n}}\left(f\left(\lambda_{n}^{2} / \Lambda^{2}\right)-\sigma^{3}\right) Y_{n}\right. \\
& \left.+\sum_{n} X_{n}^{\dagger} P_{R} \frac{1}{2 \lambda_{n}}\left(f\left(\lambda_{n}^{2} / \Lambda^{2}\right)-\sigma^{3}\right) \gamma^{\mu} \partial_{\mu} Y_{n}\right] \\
& =-\frac{i}{2} \sum_{n}\left[Y_{n}^{\dagger} \frac{1}{2}\left(1-\gamma^{5}\right)\left(f\left(\mathcal{D}^{\dagger} \not{D} / \Lambda^{2}\right)-\sigma^{3}\right) Y_{n}\right. \\
& \left.-X_{n}^{\dagger} \frac{1}{2}\left(1+\gamma^{5}\right)\left(f\left(\mathcal{D}^{\dagger} \mathcal{D}^{\dagger} / \Lambda^{2}\right)-\sigma^{3}\right) X_{n}\right] .
\end{aligned}
$$

The traces over $\sigma^{3}$ as well as the parity-even part drop out. On taking the limit of infinite regulator masses $(\Lambda \rightarrow \infty)$, the result for Euclidean signature is

$$
\begin{aligned}
\left\langle\partial_{\mu} J_{5}^{\mu}\right\rangle_{r e g} & =\lim _{\Lambda \rightarrow \infty} \frac{i}{4} \sum_{n}\left[Y_{n}^{\dagger} \gamma^{5} f\left(\mathcal{D}^{\dagger} \not \mathcal{D} / \Lambda^{2}\right) Y_{n}+X_{n}^{\dagger} \gamma^{5} f\left(\not \mathcal{D} \mathcal{D}^{\dagger} / \Lambda^{2}\right) X_{n}\right] \\
& =\frac{i \times d}{768 \pi^{2}} F_{\alpha \beta A B} \epsilon^{\alpha \beta \mu \nu} F_{\mu \nu}{ }^{A B}+\frac{i}{32 \pi^{2}} \operatorname{Tr}\left(\epsilon^{\alpha \beta \mu \nu} G_{\alpha \beta a} T^{a} G_{\mu \nu b} T^{b}\right) .
\end{aligned}
$$

$G_{\mu \nu a}$ and $F_{\mu \nu A B}$ are, respectively, the curvatures of $W_{\mu a}$ and $A_{\mu A B}$. Note that in the first line of Eq. (78) there is a factor of $\frac{1}{4}$ in the trace over $2 d$ dimensional internal space, and Dirac indices. This gives the result which is one-half of the chiral anomaly of a vector theory. Because all the fields are Weyl, the factor we get for the gravitational part is also $d$ rather than $2 d$. This is in agreement with the fact that there are $d$ Weyl fermions coupled to gravity in the bare action. 


\section{REMARKS}

We have presented a generalization of the Frolov-Slavnov invariant regularization scheme for chiral fermion theories in curved spacetimes. The Lagrangian level regularization is explicitly invariant under all the local gauge symmetries of the theory, including local Lorentz invariance. The perturbative scheme works if and only if the chiral gauge anomaly and the mixed Lorentz-gauge anomaly cancellation conditions hold. Anomalous theories manifest themselves in having divergent fermion loops which remain unregularized by the scheme. Since the invariant scheme is promoted to include local Lorentz invariance, spectator fields which do not couple to gravity cannot be, and are not, introduced. Furthermore, in the proposed scheme, the theory is truly chiral (Weyl) in that all fields are left-handed, including the regulators, and only the left-handed spin connection is needed. The scheme is therefore well suited for the study of the interaction of matter with all the four known forces in a completely chiral manner. In contrast with the vector-like formulation, the degeneracy between the ABJ current and the fermion number current in the bare action is preserved by the regularization.

How would nonperturbative effects such as global anomalies appear in the scheme? As presented, the scheme is perturbative and the success of the scheme is predicated upon the absence of perturbative gauge anomalies. A general discussion on nonperturbative effects is outside of the scope of this paper. Instead, we will focus on two ways these effects can be recognized, together with one significant consequence. For instance, it is clear that the perturbative scheme regularizes a theory with a single left-handed internal $\mathrm{SU}(2)$ doublet. Yet, it is known that such a theory suffers from the $\mathrm{SU}(2)$ global anomaly 22, 23. By embedding $\mathrm{SU}(2)$ in $\mathrm{SU}(3)$, the gauge $\mathrm{SU}(2)$ global anomaly is shown to be related to the perturbative $\mathrm{SU}(3)$ chiral gauge anomaly[24]. Within the present context, there are then fermion loops containing $\mathrm{SU}(3)$ vertices which fail to be regularized. Anomalies also manifest themselves in path integrals as nontrivial Jacobians in the measure under a change of variables 21]. From this perspective, the global SU(2) anomaly gives rise to an inconsistent Jacobian when the transformation of $(-1)$ is considered both as a $2 \pi$ rotation in $\mathrm{SU}(2)$ and as a $\pi$ rotation induced by

$\gamma^{5}$ in nontrivial $\theta$ vacua 16, 23. The present regularization scheme will not control all divergent amplitudes in these sectors. Thus a path integral for- 
mulation dependent on the tower of regulators may yield further consistency conditions from cancellation of nontrivial Jacobians. $\square$ As an example, if a Euclidean path integral is to include all topologies for four manifolds 25] and hence the required generalized spin structures 26], then a further global Lorentz anomaly cancellation condition selects grand unified theories with multiples of 16 Weyl fermions [16, 27].

Finally, it may be worthwhile to calculate the effective action generated by the theory. For instance, it is known 28] that the Einstein-Hilbert-Palatini action and the cosmological term are among the counterterms when a fermion is quantized in background curved spacetime with parity conservation. The explicitly chiral-invariant regularization scheme presented here may be used to check the resultant requisite counter terms with parity nonconservation. It is possible for example, if the torsion is not assumed to vanish, that the SamuelJacobson-Smolin [14 action of the (anti-)self-dual formulation of gravity may emerge instead from integration over the fermion and regulator fields.

\section{ACKNOWLEDGMENTS}

The research for this work has been supported by the Department of Energy under Grant No. DE-FG05-92ER40709-A005.

\section{References}

[1] S. A. Frolov and A. A. Slavnov, Phys. Lett. B296, 159 (1992).

[2] W. Pauli and F. Villars, Rev. Mod. Phys. 21, 434 (1949); S. N. Gupta, Proc. Phys. Soc. A66, 129 (1953).

\footnotetext{
${ }^{17}$ In this respect, the situation may be clearer in a truly non-perturbative formulation such as the overlap formalism for the fermion determinant [9].
} 
[3] H. Georgi, in Particles and Fields - 1974, edited by C. E. Carlson,(AIP Conf. Proc. No. 23, New York, 1975); H. Fritzsch and P. Minkowski, Ann. Phys. 93, 193 (1975).

[4] See also S. Aoki and Y. Kikukawa, Mod. Phys. Lett. A8, 3517 (1993).

[5] S. A. Frolov and A. A. Slavnov, Phys. Lett. B309, 344 (1993); Nucl. Phys. B411, 647 (1994).

[6] H. B. Nielsen and M. Ninomiya, Nucl. Phys. B183, 20 (1981); B193, 173 (1981); Phys. Lett. B105, 219 (1981).

[7] K. Okuyama and H. Suzuki, hep-th/9603062; Phys. Lett. B382, 117 (1996).

[8] K. Fujikawa, Nucl. Phys. B428, 169 (1994); in Festschrift in honor of H. Banerjee, [in Indian J. Phys. (in press)], hep-th/9506003.

[9] R. Narayanan and H. Neuberger, Phys. Lett. B302, 62 (1993).

[10] R. Narayanan and H. Neuberger, Phys. Rev. Lett. 713251 (1993); Nucl. Phys. B412, 574 (1994); B443, 305 (1995).

[11] H. T. Nieh, Phys. Rev. Lett. 53, 2219 (1984); L. Alvarez-Gaume and E. Witten, Nucl. Phys. B234, 269 (1984); S. Yajima and T. Kimura, Phys. Lett. B173, 154 (1986).

[12] L. Alvarez-Gaumé and P. Ginsparg, Nucl. Phys. B243, 449 (1984).

[13] A. Ashtekar, Phys. Rev. Lett. 57, 2244 (1986); Phys. Rev. D36, 1587(1986); New perspectives in canonical gravity, (Bibliopolis, Naples, 1988); Lectures on nonperturbative canonical gravity, (World Scientific, Singapore, 1991) and references therein.

[14] J. Samuel, Pramāna J. Phys. 28, L429(1987); Class. Quantum Grav. 5, L123(1988) ; T. Jacobson and L. Smolin, Phys. Lett. B196, 39 (1987), Class. Quantum Grav. 5, 583 (1988).

[15] A. Ashtekar, J. D. Romano and R. S. Tate, Phys. Rev. D40, 2572 (1989); T. Jacobson, Class. Quantum Grav. 5, L143 (1988); H. Kodama, Int. J. Mod. Phys. D1, 439 (1993). 
[16] L. N. Chang and C. Soo, Phys. Rev. D53, 5682 (1996).

[17] K. Fujikawa, Phys. Rev. D29 285 (1984).

[18] H. Georgi and S. Glashow, Phys. Rev. D6, 429 (1972); D. J. Gross and R. Jackiw, ibid. 6, 477 (1972); C. Bouchiat, J. Illiopoulos, and P. Meyer, Phys. Lett. 38B, 519 (1972).

[19] L. N. Chang and H. T. Nieh, Phys. Rev. Lett. 53, 21 (1984); also the second reference in [11].

[20] S. L. Adler, Phys. Rev. 177, 2426 (1969); J. S. Bell and R. Jackiw, Nuovo Cimento 60A, 47 (1969); W. A. Bardeen, Phys. Rev. 184, 1848 (1969).

[21] K. Fujikawa, Phys. Rev. D25 2584 (1982); D21, 2848 (1980); D22 1499(E) (1980); D23, 2262 (1981); Phys. Rev. Lett. 421195 (1979); ibid. 44, 1733 (1980).

[22] E. Witten, Phys. Lett. 117B, 324 (1982); Commun. Math. Phys. 100, 197 (1985).

[23] S. P. de Alwis, Phys. Rev. D32, 2837 (1985).

[24] E. Witten, Nucl. Phys. B223, 422 (1983); S. Elitzur and V. P. Nair, ibid. B243, 205 (1984).

[25] See, for instance, Euclidean Quantum Gravity, edited by G. W. Gibbons and S. W. Hawking (World Scientific, Singapore, 1993).

[26] S. W. Hawking and C. N. Pope, Phys. Lett. 73B, 42 (1978); A. Back, P. G. O. Freund and M. Forger, Phys. Lett. 77B, 181 (1978); S. J. Avis and C. J. Isham, Comm. Math. Phys. 72, 103 (1980).

[27] L. N. Chang and C. Soo, Chiral fermions, Gravity and GUTs, hepth/9411064 in Proceedings of the 4th. Drexel Symposium-1994.

[28] See, for instance, Quantum fields in curved spaces, N. D. Birrell and P. C. W. Davies (Cambridge University Press, 1982). 